\begin{tabular}{|c|c|}
\hline Title & $\begin{array}{l}\text { Robust my oelectric signal detection based on stochastic resonance using multiple surface electrode array made of } \\
\text { carbon nanotube composite paper }\end{array}$ \\
\hline Author(s) & $\begin{array}{l}\text { Shirata, Kento; Inden, Y uki; Kasai, Seiya; Oya, Takahide; Hagiwara, Y osuke; Kaeriyama, Shunichi; Nakamura, } \\
\text { Hideyuki }\end{array}$ \\
\hline Citation & $\begin{array}{l}\text { Japanese Journal of A pplied Physics(JJA P), 55(4S), 04EM07 } \\
\text { https://doi.org/10.7567/JJA P.55.04EM07 }\end{array}$ \\
\hline Issue Date & 2016-04 \\
\hline Doc URL & http:/hdl .handle.net/2115/66629 \\
\hline Rights & (C) 2016 The Japan Society of A pplied Physics \\
\hline Type & article (author version) \\
\hline File Information & |JJAP2015_55_SS15225.pdf \\
\hline
\end{tabular}

Instructions for use 


\section{Robust myoelectric signal detection based on stochastic resonance using multiple-surface-electrode array made of carbon nanotube composite paper}

Kento Shirata $^{1}$, Yuki Inden ${ }^{1}$, Seiya Kasai ${ }^{1 *}$, Takahide Oya ${ }^{2}$, Yosuke Hagiwara ${ }^{3}$, Shunichi Kaeriyama $^{3}$, and Hideyuki Nakamura ${ }^{3}$

${ }^{1}$ Research Center for Integrated Quantum Electronics and Graduate School of Information Science and Technology, Hokkaido University, Sapporo 060-8628, Japan

${ }^{2}$ Graduate School of Engineering, Yokohama National University, Yokohama 240-8501, Japan

3 The Semiconductor Technology Academic Research Center (STARC), Yokohama 222-0033, Japan

*E-mail: kasai@rciqe.hokudai.ac.jp

We investigated the robust detection of surface electromyogram (EMG) signals based on the stochastic resonance (SR) phenomenon, in which the response to weak signals is optimized by adding noise, combined with multiple surface electrodes. Flexible carbon nanotube composite paper (CNT-cp) was applied to the surface electrode, which showed good performance that is comparable to that of conventional $\mathrm{Ag} / \mathrm{AgCl}$ electrodes. The SR-based EMG signal system integrating an 8-Schmitt-trigger network and the multiple-CNT-cp-electrode array successfully detected weak EMG signals even when the subject's body is in the motion, which was difficult to achieve using the conventional technique. The feasibility of the SR-based EMG detection technique was confirmed by demonstrating its applicability to robot hand control. 


\section{Introduction}

Recently, man-machine interfaces (MMIs) have come to play a key role in various types of electronic equipment. For user-friendly MMIs achieving intuitive machine control, detection and use of surface electromyogram (EMG) signals have attracted much attention. $^{1-3)}$ EMG signals are trains of voltage pulses generated by the activity of muscles and it includes information of the motion of the human body. ${ }^{4-8)}$ Surface EMG signals, which can be taken noninvasively, are necessary for MMI applications. Surface EMG signals are attenuated significantly on the body surface ${ }^{9,10)}$ and their amplitudes range from $10 \mu \mathrm{V}$ to $10 \mathrm{mV}$. Thus, surface EMG signals are usually contaminated by noise and their detection is often difficult. In a conventional EMG detection technique called as bipolar lead, EMG signals are amplified using a differential amplifier with two surface electrodes. When the two electrodes are attached at the appropriate positions, noises induced on the two electrodes are in the same phase whereas the surface EMG signals are in the anti phase. Then the differential amplifier can cancel out the noise and amplify the EMG signals. This means that the bipolar lead is quite sensitive to the contact balance between the two electrodes. When the motion of the subject causes fluctuations of the contacts, the system inevitably amplifies the noise and cancels the signals, and the output signal-to-noise ratio (SNR) decreases drastically. This is a problem in the application of EMG to MMIs for daily use that would allow easy attachment to the body of a subject.

As an alternative surface EMG signal detection technique, we have developed a novel one based on the stochastic resonance (SR) phenomenon. SR is a nonlinear phenomenon in which a response to a weak signal is optimized or improved by adding noise. ${ }^{11-22)}$ This phenomenon is often seen in the sensory functions of various biological systems. ${ }^{23-25)}$ In addition, the biological systems often enhance the SR response by forming summing networks with many sensors in parallel. ${ }^{26)}$ We already confirmed that the SR-based technique can achieve a higher SNR than the conventional technique. ${ }^{27)}$ The purpose of this study is to investigate a robust EMG signal detection technique by combining SR and a flexible multiple surface electrode array to achieve both the increase in sensitivity and elimination of contact fluctuation. We introduce the novel use of carbon nanotube composite paper (CNT-cp) as the surface electrode material, which 
is flexible and easy to handle. We characterize the performance of the CNT-cp-based surface electrode. Then, the SR-based EMG detection system with a multiple-surface-electrode array is implemented and characterized.

\section{Concept and implementation}

The basic concept of the surface EMG detection system is shown in Fig. 1(a). Our system achieves high SNR and robustness against the motion of the body owing to the application of the SR phenomenon together with the multiple surface electrode array. The multiple-surface-electrode configuration can eliminate contact fluctuation by averaging. Figure 1(b) shows the calculated output SNRs of the bipolar lead and the standard averaging technique. For simplicity, we assume that the input SNR is 1 and uncorrelated noise is superimposed on each electrode in the multiple-surface-electrode array. In this calculation, half of the electrodes have imperfect contacts. Contact balance in this figure corresponds to the input amplitude ratio of the imperfect to perfect contacts. In the case of the multiple electrodes, the response becomes increasingly robust against the contact unbalance with increasing number of electrodes. The output SNR of the multiple electrodes is better than that of the bipolar lead when the contacts are unbalanced. A typical response of SR and its mechanism is shown in Fig. 1(c). The basic mechanism of SR involves noise-assisted state transition in a nonlinear system. ${ }^{11)}$ When an input signal is weak, the signal cannot cross the threshold and no response is obtained in the output. On the other hand, when a moderately strong noise is added to the input signal, the noise-added signal crosses the threshold stochastically and the response appears in the output. Here, the input and output waveforms clearly correlate. Note that the amplitude of the input signal is successfully encoded to pulse density or pulse width in the output owing to the nonlinear operation of the device. The original input can be reproduced by decoding the pulse train using a low-pass filter. ${ }^{28)}$ Threshold and bistable systems are often used to electrically induce SR, such as a comparator and a Schmitt trigger. ${ }^{29,30)}$

To enhance the SR response, formation of a summing network as shown on the right side of Fig. 1(a) is effective. ${ }^{26)}$ This network integrates many SR devices in parallel, whose configuration is based on the sensory neurons in biological systems. A 
common input signal is given to all devices and the outputs of the devices are summed up. If an uncorrelated noise is imposed on all the devices, the summed output successfully reproduces the original input signal. ${ }^{26)}$ Our EMG detection system merges this summing network of the SR devices with the multiple surface electrode array. In this system, each electrode is connected to an SR device. When the contact fluctuation caused by body motion arises in several electrodes, different noises are imposed on them. This corresponds to the situation that an uncorrelated noise is imposed on all the SR devices, which positively contributes to the reproduction of the original input signal. Our system uses internal noise to cause SR without adding external noise. Thus, a noise source is not necessary for this system. We adjust the system to obtain the peak of the SR response by tuning the threshold values. In addition, massive integration of SR devices can maintain high input-output correlation even when the noise intensity changes.

For the surface electrodes for EMG detection, $\mathrm{Ag} / \mathrm{AgCl}$ with electrolyte paste is commonly used. However, such electrodes are inflexible and contact fluctuation takes place when the subject moves. In this study, we investigate the use of carbon nanotube-composite paper (CNT-cp). A surface electrode needs to be sensitive to EMG signals and low-intensity noise. It must be harmless to humans and flexible for stable contact to the skin on the curved parts of the body. CNT-cp satisfies almost all these requirements, except for its relatively high sheet resistance. This material is also attractive for easy fabrication of multiple electrodes by cut and paste. It is softer and warmer on the skin than metal electrodes.

\section{Experimental procedure}

Prior to the examination of the SR-based EMG signal detection system, we characterized the CNT-cp surface electrodes for EMG signal detection. Figure 2 shows photographs of a CNT-cp electrode and a commercially available conventional $\mathrm{Ag} / \mathrm{AgCl}$ electrode, together with a diagram of the unipolar lead for the electrode characterization. Both of them were circular in shape and their diameters were both $8 \mathrm{~mm}$. CNT-cp was prepared by mixing multiwalled carbon nanotubes (MWNTs) into a piece of paper. ${ }^{31,32)}$ The thickness of the paper was $50 \mu \mathrm{m}$ and the CNT content was $0.5 \mathrm{~g} / \mathrm{cm}^{2}$. The surface 
color of the CNT-cp reflected the amount of the mixed CNTs. The sheet resistance was $40 \Omega /$ sq. We used the unipolar lead technique for the characterization of the electrodes, in which the EMG signal induced in the surface electrode was amplified after filtering DC and $50 \mathrm{~Hz}$ hum noise. The surface electrodes were fixed on the ulnar forearm of a subject using an adhesive tape. An EMG signal was generated by tensing the forearm muscle by making a fist, without any motion of the body of the subject. The effect of the electrolytic paste on EMG signal detection was also examined.

The SR-based EMG signal detection system is shown in Fig. 3. It integrated 8 Schmitt triggers as bistable nonlinear devices in parallel. A Schmitt trigger was formed using an operational amplifier with a feedback. It had hysteresis characteristic with two thresholds. Its hysteresis width could be adjusted by changing a variable resistor in the circuit. The EMG signal induced in each surface electrode was fed into an active high-pass filter (HPF) ${ }^{33)}$ and a band elimination filter (BEF) for canceling the DC offset and filtering the $50 \mathrm{~Hz}$ hum noise, respectively. Then the filtered signal, which still included high-frequency noise, was inputted into the Schmitt trigger. The output of the system was obtained by summing up the outputs of all the Schmitt triggers using a summing circuit. The system output waveforms were recorded using a digital oscilloscope. The system above was implemented using the surface mount technique. The circuit board size was $4 \times 5 \mathrm{~cm}^{2}$. The system was operated at a dual supply voltage of $V_{\mathrm{dd}}=5 \mathrm{~V}$ and $V_{\mathrm{ss}}=-5 \mathrm{~V}$. The evaluated power consumption was $70 \mathrm{~mW}$. This system did not implement any noise source. Unintentionally imposed noise on the EMG signal was considered as noise that causes SR. Instead of adjusting noise intensity, we adjusted the hysteresis width of each Schmitt trigger so as to clearly obtain the EMG signal. The adjusted hysteresis width was typically $0.1 \mathrm{~V}$ in all the Schmitt triggers. For the multiple surface electrode array, eight CNT-cp electrodes were arranged in 2 rows x 4 columns. The column of the electrodes was aligned along the forearm. Each electrode were circular in shape with $8 \mathrm{~mm}$ diameter. The pitch of the electrodes were $11 \mathrm{~mm}$. The electrode array was easily attached onto the ulnar forearm surface using an arm band without using an electrolytic paste or an adhesive tape, which made it possible to easily attach the multiple electrode array onto the body of the subject. We examined the effect of body motion on EMG signal detection. Body motion was performed by moving the 
upper arm forward and backward.

\section{Results and discussion}

\subsection{CNT-cp surface electrodes}

Figure 4 shows the waveforms of the EMG signals obtained using the conventional $\mathrm{Ag} / \mathrm{AgCl}$ and CNT-cp electrodes with and without the electrolytic paste. The $\mathrm{Ag} / \mathrm{AgCl}$ electrodes exhibited dense pulse trains when tensing the forearm by clenching a fist, as shown in Fig. 4(a). The $\mathrm{Ag} / \mathrm{AgCl}$ electrodes with and without the paste achieved SNRs of 18 and $16 \mathrm{~dB}$, respectively. The electrolytic paste increased the SNR as expected. The CNT-cp electrode could also detect EMG signals clearly, as shown in Fig. 4(b). The evaluated SNRs with and without the paste were 20 and $17 \mathrm{~dB}$, respectively. These were comparable to that of the $\mathrm{Ag} / \mathrm{AgCl}$ electrode with the paste. The CNT-cp electrode showed high sensitivity even without the paste. In general, a low impedance is necessary for the surface electrode to detect EMG signals with small loss. ${ }^{34)}$ However, the sheet resistance of the CNT-cp electrode was 100 times higher than that of the $\mathrm{Ag} / \mathrm{AgCl}$ and the obtained result seemed to contradict the requirement of the surface electrode.

We considered that the good performance of the CNT-cp electrode in the surface EMG signal detection was attributed to the low contact resistance to the surface skin. Then, we estimated contact resistance by measuring the current between the two surface electrodes attached onto the skin surface of the forearm at an applied voltage of $1 \mathrm{~V}$. The interval of the electrodes was $2 \mathrm{~cm}$. We also characterized a CNT-coated polyethylene terephthalate (PET) film with a smooth surface and a platinum (Pt) film with a very low sheet resistance. The evaluated contact resistances are summarized in Table I. The measured resistances were on the $M \Omega$ order and they were much higher than the resistances of the electrode and skin: less than $10 \Omega$ and $2-4 \mathrm{k} \Omega$, respectively Therefore, the contact resistance between the electrode and the skin predominated the measured resistance. The evaluated contact resistance of the conventional $\mathrm{Ag} / \mathrm{AgCl}$ electrode with the electrolytic paste was $1 \mathrm{M} \Omega$, whereas those of the CNT-cp with and without the paste were 0.5 and $1.3 \mathrm{M} \Omega$, respectively. The Pt electrode showed the highest resistance among the examined electrodes even for those with the lowest sheet resistance. The low contact resistance is a possible reason for the observed good 
performance of the CNT-cp electrode. On the other hand, the Pt and CNT PET films having smooth surfaces showed higher contact resistances than other electrodes, suggesting that the resistance depended on the texture of the electrode surface. We measured the surface roughness of the electrodes and the results are also shown in Table I. The evaluated root mean square (RMS) of the measured surface roughness was $14 \mu \mathrm{m}$ for the $\mathrm{Ag} / \mathrm{AgCl}$ electrode, $21 \mu \mathrm{m}$ for the CNT-cp electrode, $5 \mu \mathrm{m}$ for the CNT film, and $0.5 \mu \mathrm{m}$ for $\mathrm{Pt}$ film. It was found that the rough surface helped reduce contact resistance. Skin keratin has roughly $20 \mu \mathrm{m}$ roughness, comparable to that of the CNT-cp electrode. Considering that the CNT film showed a relatively high contact resistance even with its flexibility, the texture of the paper played a major role in decreasing contact resistance; the corrugated texture of the CNT-cp electrode increased the area of contact with the skin surface. Figure 5 shows the measured contact resistance change for the CNT-cp and Pt film electrodes on the skin as a function of the pressure on the electrodes. The contact resistance of the CNT-cp electrode decreased after applying pressure, whereas that of the Pt film hardly changed. The obtained results indicated that the pressure made the CNT-cp electrode mesh with the skin surface, resulting in the increase in the effective contact area between the CNT-cp electrode and the skin surfaces.

\subsection{Signal detection circuit}

Figure 6(a) shows the forearm EMG waveforms in the resting state of the subject obtained using unipolar lead, bipolar lead, and SR-based techniques. The output of the unipolar lead always contained noise and the evaluated output SNR was $20 \mathrm{~dB}$. The bipolar lead successfully reduced such noise and the output SNR of $28 \mathrm{~dB}$ was obtained. The SNR of the SR-based technique could achieve the highest SNR of $40 \mathrm{~dB}$ among the three techniques. This was because the SR-based technique could eliminate the noise even in the same frequency band of the EMG signal by the nonlinear transfer function. ${ }^{35)}$ The amplitudes of the output in the SR-based technique were almost the same and the technique appeared to lose information of the original EMG signal. However, the intensity of the EMG signal was converted to the width and density of the pulses owing to the nonlinear transfer curve of the Schmitt trigger. In addition, the 
detailed amplitude of each pulse had multiple values in accordance with the input amplitude. We separately confirmed that the output pulse trains maintained the information of the input EMG signal.

Figure 6(b) shows the forearm EMG signals obtained during the motion of the upper arm. The tension of the muscle could be identified from the output of the unipolar lead, although the waveform fluctuated throughout the time when the upper arm was in motion. The evaluated output SNR was markedly decreased to $7 \mathrm{~dB}, 13 \mathrm{~dB}$ lower than that without the motion of the upper arm. The output of the bipolar lead always markedly fluctuated regardless of the tension of the muscle, which arose from the imbalance of two electrode contacts. It was impossible to distinguish the muscle tension from the noisy output waveform and we could not evaluate the output SNR. On the other hand, the output of the SR-based technique could respond to muscle tension even during the motion of the upper arm and achieved a high output SNR of $20 \mathrm{~dB}$. The obtained results confirmed the robustness and high sensitivity of our SR-based technique of detecting surface EMG signals during body motion.

\subsection{Application to machine control}

We demonstrated the feasibility of our SR-based technique for MMIs with robot hand control using the surface EMG signals on the forearm of the subject. A diagram and a photograph of the robot hand control system are shown in Fig. 7. The EMG signals on the forearm were obtained using the SR-based technique, which was the same as that in Fig. 3. From the EMG signals, control commands of hand opening or grasping were estimated using a simple decision algorithm implemented on an on-board microcomputer. The robot hand grasped when the muscle of the forearm tensed and it opened when the muscle was relaxed. We were able to successfully control the robot hand regardless of the motion of the upper arm (see supplementary). On the other hand, the system using the conventional bipolar lead lost the control of the robot hand during the motion of the upper arm. The obtained results confirmed the feasibility of the SR-based EMG signal detection technique for MMIs used in daily life where the body of the user moves frequently. 


\section{Conclusions}

We investigated the robust detection of EMG signals based on the SR phenomenon using multiple surface electrodes. CNT-cp was applied to the surface electrodes and the electrodes showed good performance comparable to that of conventional $\mathrm{Ag} / \mathrm{AgCl}$ electrodes. The SR-based EMG signal detection system integrating an 8-Schmitt-trigger network and multiple CNT-cp electrodes successfully detected weak EMG signals even the subject's body is in motion. The feasibility of the SR-based technique was confirmed by demonstrating its applicability to robot hand control. The obtained results suggest a good possibility of our system for application to MMIs used in daily life.

\section{Acknowledgements}

The authors thank KJ Specialty Paper Co., Ltd., for providing CNT-based materials. This work is partly supported by the STARC program and a Grant-in-Aid for Scientific Research on Innovative Areas "Molecular Architectonics" (No. 25110001) from the Ministry of Education, Culture, Sports, Science and Technology, Japan.

\section{References}

1) R. de la Rosa, A. Alonso, A. Carrera, R. Durán, and P. Fernández, Sensors 10, 11100 (2010).

2) C. Castellini and P. van der Smagt, Biol. Cybern. 100, 35 (2009).

3) K. Kiguchi, M. H. Rahman, M. Sasaki, and K. Teramoto, Rob. Auton. Syst. 56, 678 (2008).

4) M. Atzori, A. Gijsberts, C. Castellini, B. Caputo, A. Mittaz Hager, S. Elsig, G. Giatsidis, F. Bassetto, and H. Müller, Sci. Data 1, 140053 (2014).

5) L. H. Lindström and R. I. Magnusson, Proc. IEEE 65, 653 (1977).

6) H. J. Hermins, B. Freriks, C. Disselhorst-Klug, and G. Rau, J. Electromyogr. Kinesiology. 10, 361 (2000).

7) M. B. I. Reaz, M. S. Hussain, and F. Mond-Yasin, Biol. Proced. Online 8, 11 (2006).

8) A. Phinyomark, P. Phukpattaranont, and C. Limsakul, Expert Syst. Appl. 39, 7420 
(2012).

9) J. C. Rothwell, J. Neurosci. Methods 74, 113 (1997).

10) D. Farina, R. Merletti, and Roger M. Enoka, J. Appl. Physiol. 96, 1486 (2004).

11) L. Gammaitoni, P. Hänggi, P. Jung, and F. Marchesoni, Rev. Mod. Phys. 70, 223 (1998).

12) R. Benzi, A. Sutera, and A. Vulpiani, J. Phys. A 14, L453 (1981).

13) D. Petracchi, Chaos Solitons Fractals 11, 1827 (2000).

14) K. Loerincz, Z. Gingl, and L. B. Kiss, Phys. Lett. A 224, 63 (1996).

15) M. Misono, T. Kohmoto, Y. Fukuda, and M. Kunitomo, Opt. Commun. 152, 255 (1998).

16) S. Kasai and T. Asai, Appl. Phys. Express 1, 083001 (2008).

17) S. Kasai, K. Miura, and Y. Shiratori, Appl. Phys. Lett. 96, 194102 (2010).

18) S. Kasai, Y. Shiratori, K. Miura, Y. Nakano, and T. Muramatsu, Phys. Status Solidi C 8, 384 (2011).

19) T. Oya, T. Asai, and Y. Amemiya, Chaos Solitons Fractals 32, 855 (2007).

20) F. Moss, L. M. Ward, and W. G. Sannita, Clin. Neurophysiol. 115, 267 (2004).

21) G. P. Harmer and D. Abbott, Microelectronics J. 31, 533 (2000).

22) D. Nozaki, D. J. Mar, P. Grigg, and J. J. Collins, Phys. Rev. Lett. 82, 2402 (1999).

23) D. F. Russell, L. A. Wilkens, and F. Moss, Nature 402, 291 (1999).

24) J. E. Levin and J. P. Miller, Nature 380, 165 (1996).

25) K. Wiesenfeld and F. Moss, Nature 373, 33 (1995).

26) J. J. Collins, C. C. Chow, and T. T. Imhoff, Nature 376, 236 (1995).

27) Y. Imai, M. Sato, T. Tanaka, S. Kasai, Y. Hagiwara, H. Ishizaki, S. Kuwabara, and T. Arakawa, Jpn. J. Appl. Phys. 53, 06JE01 (2014).

28) Y. Tadokoro, S. Kasai, and A. Ichiki, Digital Signal Process. 37, 1 (2015).

29) Z. Gingl, R. Vajtai, and L.B. Kiss, Chaos Solitons Fractals 11, 1929 (2000).

30) S. Fauve and F. Heslot, Phys. Lett. A 97, 5 (1983).

31) Y. Fujitsuka and T. Oya, J. Nanotechnol. 2012, 819281 (2012).

32) T. Oya and T. Ogino, Carbon 46, 169 (2010).

33) C. J. De Luca, L. D. Gilmore, M. Kuznetsov, and S. H. Roy, J. Biomech. 43, 1573 (2010).

34) E. Huigen, A. Peper, and C. A. Grimbergen, Med. Biol. Eng. Comput. 40, 332 
(2002).

35) S. Kasai, Y. Tadokoro, and A. Ichiki, Phys. Rev. E 88, 062127 (2013).

\section{Table caption}

Table I Surface electrode materials and their characteristics

\section{Figure captions}

Fig. 1 Basic concept of SR-based EMG signal detection: (a) system diagram, (b) calculated output SNR as a function of contact balance for bipolar lead and multiple electrodes, and (c) mechanism of SR in bistable system.

Fig. 2 Photograph of CNT composite paper and conventional $\mathrm{Ag} / \mathrm{AgCl}$ surface electrodes. The diagram of the unipolar lead for signal detection is also shown.

Fig. 3 Implemented SR-based surface EMG signal detection system.

Fig. 4 EMG waveforms obtained using (a) $\mathrm{Ag} / \mathrm{AgCl}$ and (b) CNT-cp electrodes with and without electrolytic paste.

Fig. 5 Change in contact resistance on the skin surface as a function of pressure applied on CNT-cp and Pt thin film surface electrodes.

Fig. 6 Surface EMG signals on forearm obtained using unipolar lead, bipolar lead, and SR-based system (a) without and (b) with motion of the upper arm.

Fig. 7 Diagram of a EMG-based robot hand control system operated using the SR-based EMG detection technique and photograph of the system. 


\section{Table I Kento Shirata et al.}

\begin{tabular}{|c|c|c|c|c|}
\hline Material & Paste & $\begin{array}{c}\text { Sheet } \\
\text { Resistance }\end{array}$ & $\begin{array}{c}\text { Contact } \\
\text { Resistance }\end{array}$ & $\begin{array}{c}\text { Surface } \\
\text { roughness }\end{array}$ \\
\hline $\begin{array}{c}\text { Ag/AgCl } \\
\text { (conventional) }\end{array}$ & coat & $0.5 \Omega / \square$ & $1.0 \mathrm{M} \Omega$ & \multirow{2}{*}{$14 \mu \mathrm{m}$} \\
\hline \multirow{2}{*}{ CNT-cp } & non & \multirow{2}{*}{$40 \Omega / \square$} & $1.3 \mathrm{M} \Omega$ & \multirow{2}{*}{$21 \mu \mathrm{m}$} \\
\cline { 2 - 2 } & Coat & & $0.5 \mathrm{M} \Omega$ & \\
\hline \multirow{2}{*}{ CNT film } & non & \multirow{2}{*}{$52 \Omega / \square$} & $5.6 \mathrm{M} \Omega$ & \multirow{2}{*}{$5 \mu \mathrm{m}$} \\
\cline { 2 - 2 } & coat & & $4.4 \mathrm{M} \Omega$ & \multirow{2}{*}{$0.5 \mu \mathrm{m}$} \\
\hline \multirow{2}{*}{ Pt film } & non & \multirow{2}{*}{$5 \mathrm{~m} \Omega / \square$} & $9.2 \mathrm{M} \Omega$ & \multicolumn{2}{|c|}{$5.9 \mathrm{M} \Omega$} & \\
\cline { 2 - 2 } & coat & & \multicolumn{2}{|c}{} \\
& & &
\end{tabular}




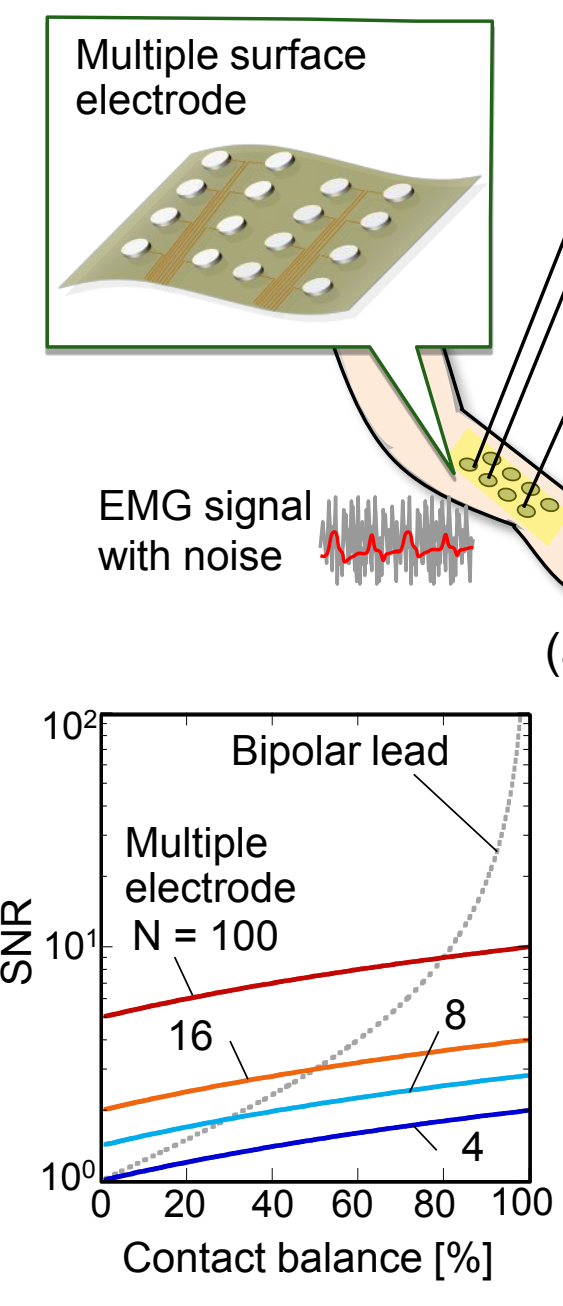

(b) (a)
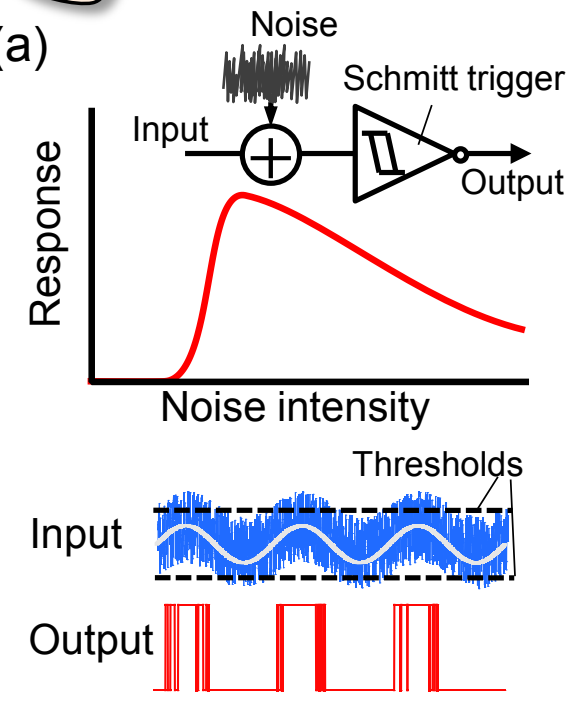

(c)

Fig. 1 Kento Shirata et al. 


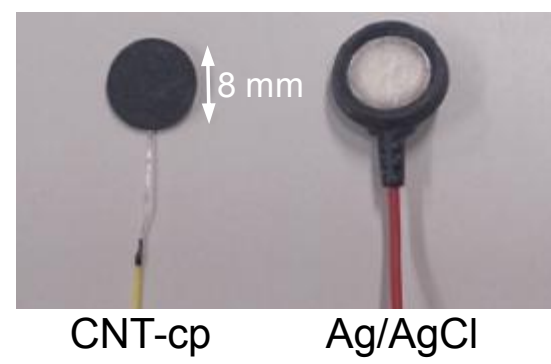

Surface electrode

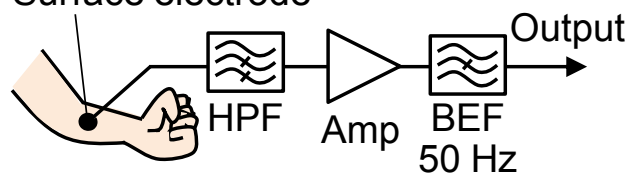

Fig. 2 Kento Shirata et al. 


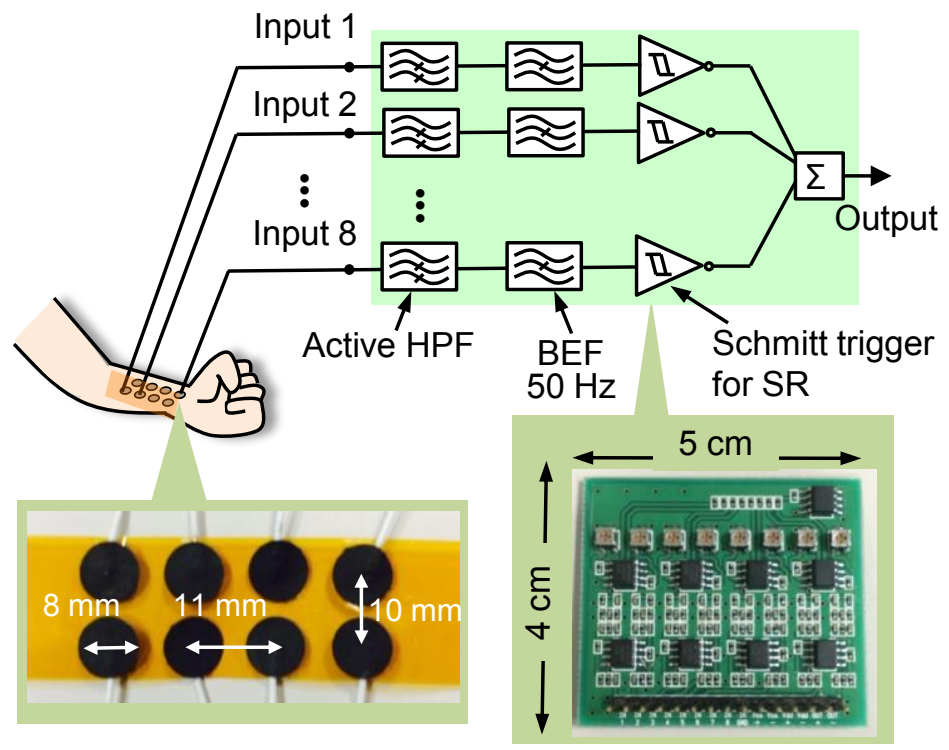

Fig. 3 Kento Shirata et al. 
(a)

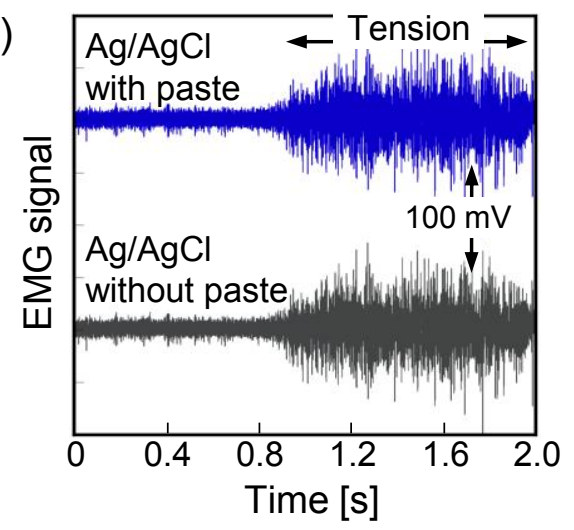

(b)

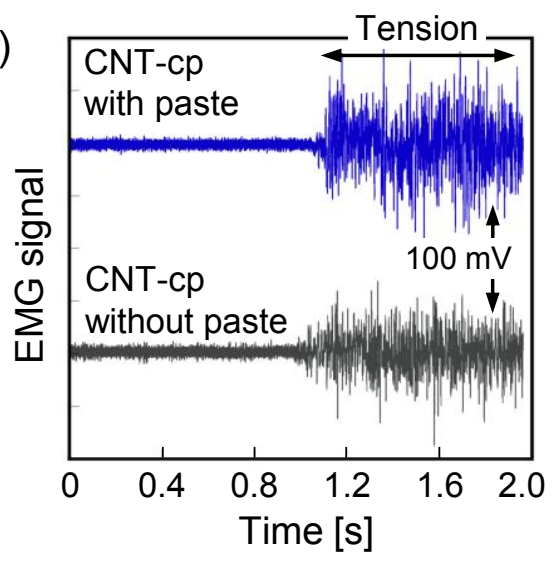

Fig. 4 Kento Shirata et al. 


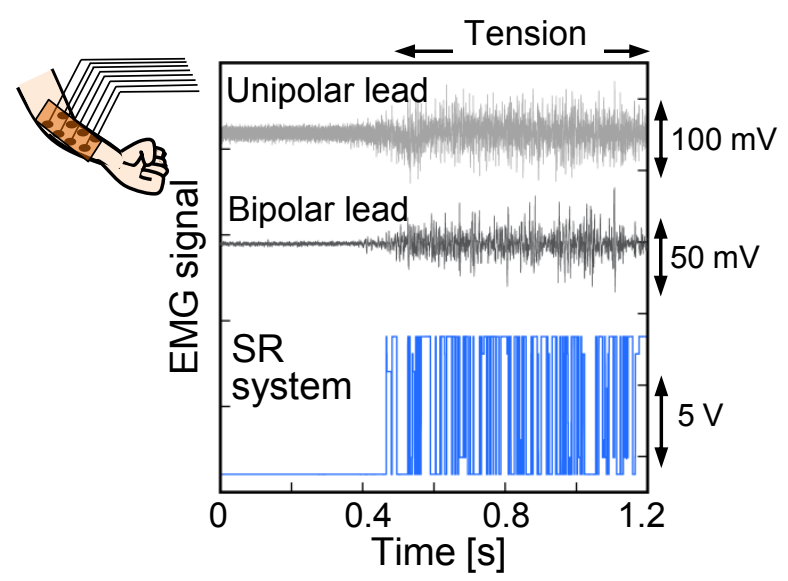

(a)

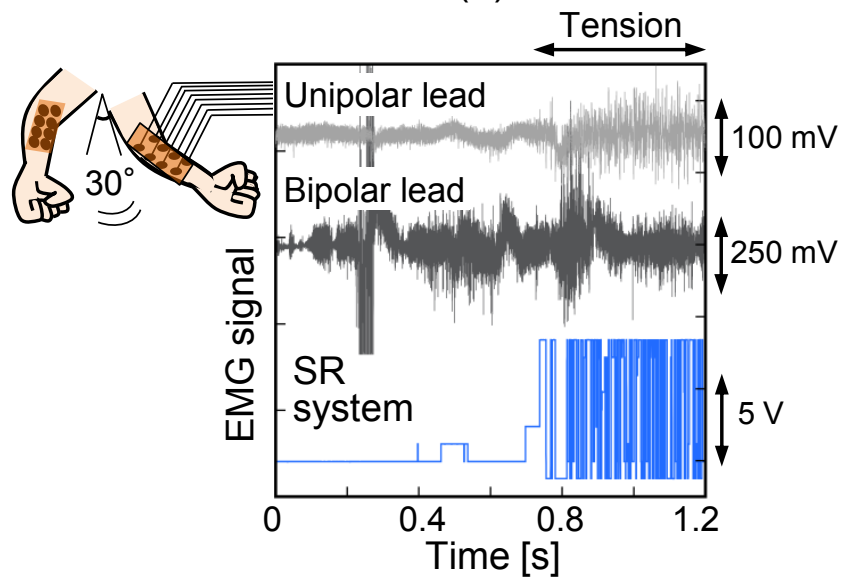

(b)

Fig. 5 Kento Shirata et al. 

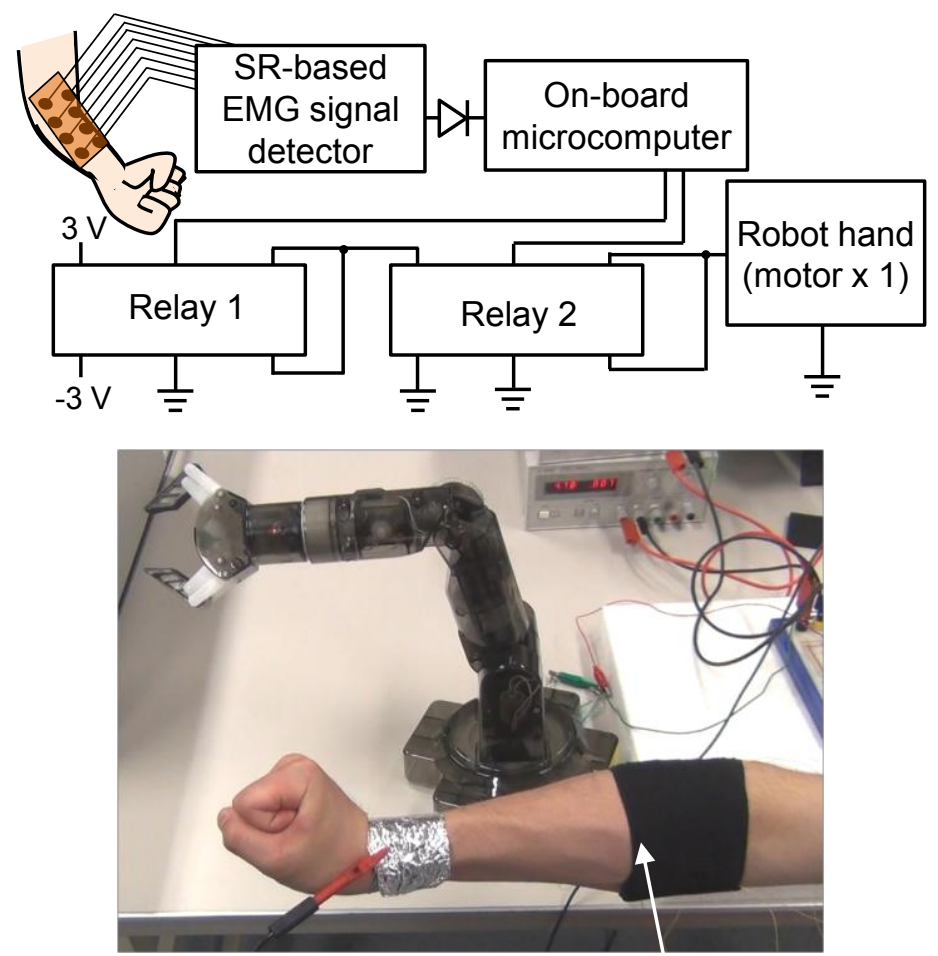

Multiple surface electrodes

fixed by arm band

Fig. 6 Kento Shirata et al. 